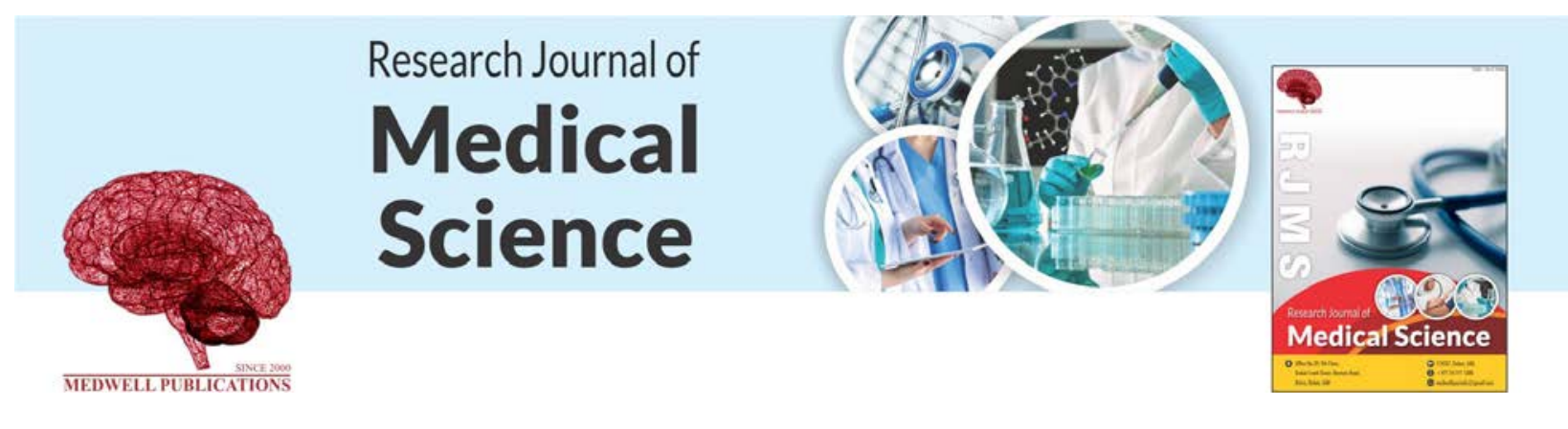

\title{
Updates of Coronavirus: Basic and Clinical Investigations
}

\author{
${ }^{1}$ Ahed J. Alkhatib, ${ }^{2}$ Rabaa Y. Athamneh and ${ }^{3}$ Rawan F. Ghazw \\ ${ }^{1}$ Department of Legal Medicine, Toxicology of Forensic Science and Toxicology, School of Medicine, Jordan \\ University of Science and Technology, Irbid, Jordan \\ ${ }^{2}$ Department of Medical Microbiology and Clinical Microbiology, Near East University, Nicosia \\ ${ }^{3}$ Department of Pharmacology, Faculty of Medicine, Jordan University of Science and Technology, Irbid, \\ Jordan
}

Key words: Coronaviruses, COVID-19, RNA, fever, cough

Corresponding Author:

Ahed J. Alkhatib

Department of Medicine and Critical Care, International Mariinskaya Academy

Page No.: 54-57

Volume: 14, Issue 3, 2020

ISSN: $1815-9346$

Research Journal of Medical Sciences

Copy Right: Medwell Publications
Abstract: The present study aimed to review the literature regarding Coronavirus disease (COVID-19). Basic and clinical updates were reviewed. Coronaviruses are infections of both clinical and veterinary significance. Coronaviruses are single stranded RNA infections with a lipoprotein envelope and enter the host cells by a Class I combination protein, for example, one that doesn't require some other viral surface proteins for combination. Clinical presentation of patients with Coronaviruses included cough, fever, headache. The age of patients in some cases was as early as 112 days. As a conclusion, although, the existence of widespread of Coronaviruses, vital information remains to be elucidated.

\section{INTRODUCTION}

An overview of Coronaviruses: Coronaviruses are infections of both clinical and veterinary significance ${ }^{[1]}$. They incorporate Transmissible Gastroenteritis Virus (TGEV), porcine pestilence looseness of the Porcine Epidemic Diarrhea Virus (PEDV) and the human Coronaviruses severe acute respiratory syndrome Coronavirus (SARS-CoV) liable for the pestilence in 2003 and Middle East respiratory syndrome Coronavirus $(\mathrm{MERS}-\mathrm{CoV})^{[2]}$.

In the previous 10 years, numerous new Coronaviruses have been recognized. They contaminate a wide scope of hosts from warm blooded creatures to winged creatures and firmly related Coronaviruses have been recognized in indirectly related creatures recommending ongoing interspecies bounces ${ }^{1]}$. Like flu infections that have comparable pestilence properties, they are single stranded RNA infections with a lipoprotein envelope and enter the host cells by a Class I combination protein, for example one that doesn't require some other viral surface proteins for combination. Viral combination proteins are potential helpful and immunization targets and the above is demonstrative of the likelihood that the sort of contemplations examined in the current paper can be stretched out to more extensive scope of viruses ${ }^{[2]}$.

The Coronavirus genome of intrigue, varieties and configuration issues: In spite of the above remarks, it remains that in the beginning times of a pandemic, one needs to guarantee that one is tending to the infection portrayed and in fact be wary about what the expression "a similar infection" might mean by. In such manner, just as a matter of writing looking, it has helped neither specialists nor news associations (and meeting and gathering coordinators) that the name for the infection and malady has continually changed in the course of recent 
weeks and for sure days. The WHO chose to lean toward the name "2019-nCoV" hours prior arrival of the past report by the current creator in January 2020, in spite of the fact that a ultimate conclusion on the infection's authentic name despite everything anticipated the Universal Committee on Taxonomy of Viruses. At the hour of last composing of this content, the WHO named the malady COVID-19, so one can sensibly talk about the "COVID-10" or "Covid-19 infection". The above mentioned past report by the creator was right on time in bringing up that the (Wuhan fish advertise disconnect) infection surface protein specifically noteworthy beneath is firmly identified with that of human and bat SARS and at the time of changing the current paper, the Coronavirus Study Group (CSG) of the International Committee on Taxonomy of Viruses concluded that 2019-nCoV was a variation of the coronavirus that caused an episode of serious intense respiratory disorder (SARS) in 2002-03. Anyway the new name SARS-CoV-2 isn't all around well known and the classification 2019-nCoV infection or only 2019-nCoV is utilized all through this content. Chinese specialists and government have worked productively to distribute their assurance of the early identified Wuhan infection on a few sites. At the hour of composing, there were a few concerns communicated, regularly outside the standard friend checked on logical writing, that what is viewed as the current 2019-nCoV pandemic isn't because of a similar infection as that determined as "Wuhan fish show case pneumonia infection seclude", for example, GenBank section MN908947 which is utilized here. On January 17, 2019, MN908947.3 supplanted MN908947.2 and likely speaks to a satisfactory stable portrayal of the arrangement for examination into that strain seclude. The passage portrays a RNA infection with a RNA grouping of 29033 bases. Regardless of the above concerns, it is accepted that MN908947.3, accessible at the hour of the current examination is basically the Wuhan fish advertise segregate and 2019-nCoV with the exception of any genome changes because of acknowledged transformations as is ordinarily the situation in the course of a viral plague. RNA infections normally held to have a lot higher change rates than DNA infections. It is portion of the outside of an infection that regularly connects with the host cells for passage and which are powerless against the host safe framework. Tragically, it is likewise uncovered locales on surface proteins that acknowledge transformations all the more promptly with the exception of at significant cooperation destinations. In the event that mixes structured as weapons against the infection depend on segments of amino corrosive grouping that can promptly change, at that point they will immediately turn into pointless ("escape by transformation”). The strain is along these lines again significant in any case, as noted above, it is no less the varieties between infection genomes, taking into account the connections of the genome of enthusiasm with those of both firmly related and remotely related infections that are especially, useful for the current sort of study. This is likewise significant on the grounds that direct data about the structure, capacity and activity of the proteins encoded by the genome isn't, at time of composing, accessible for 2019-nCoV and one tries to make sensible extrapolations from what is known for other related infections ${ }^{[2]}$.

Lai et $a l^{[3]}$ conducted a study in the light of considerations such as the development of serious severe acute respiratory syndrome Coronavirus 2 (SARS-CoV-2; already temporarily named 2019 novel coronavirus or 2019-nCoV) ailment (COVID-19) in China toward the end of 2019 has caused an enormous worldwide episode and is a significant general medical problem. Starting at 11 February 2020, information from the World Health Organization (WHO) have demonstrated that in excess of 43000 affirmed cases have been recognized in 28 nations/areas with $>99 \%$ of cases being distinguished in China. On 30 January 2020, the WHO announced COVID-19 as the 6 th general wellbeing crisis of universal concern. SARS-CoV-2 is firmly identified with two batinferred extreme severe respiratory syndrome like coronaviruses, bat-SL-CoVZC45 what's more, bat-SLCoVZXC21. It is spread by human-to-human transmission through beads or direct contact and disease has been evaluated to have mean hatching time of 6.4 days and an essential multiplication number of 2.24-3.58. Among patients with pneumonia brought about by SARS-CoV-2 (novel coronavirus pneumonia or on the other hand Wuhan pneumonia), fever was the most widely recognized manifestation, trailed by hack. Respective lung association with ground-glass obscurity was the most widely recognized finding from processed tomography pictures of the chest. The one instance of SARS-CoV-2 pneumonia in the USA is reacting great to remdesivir, which is currently experiencing a clinical preliminary in China. At present, controlling contamination to forestall the spread of SARS-CoV-2 is the essential intercession being utilized. Be that as it may, general wellbeing specialists should continue observing the circumstance intently, as the more we can find out about this novel infection and its related episode, the better, we can react. Interactions between human and animals regarding Coronavirus.

Albeit early examinations revealed a connection between a solitary nearby fish and wild creature showcase and most instances of contamination, demonstrating conceivable creature to-human transmission, contemplates have progressively exhibited human-to-human transmission of SARS-CoV-2 through beads or direct 
contact $^{[4-7]}$. In addition, as indicated by one examination, assumed medical clinic related transmission of SARS-CoV-2 was associated in $41 \%$ with patients ${ }^{[7]}$. In view of the proof of a quickly expanding occurrence of contaminations $^{[8]}$ and the chance of transmission by asymptomatic transporters ${ }^{[9]}$, SARS-CoV-2 can be transmitted viably among people and displays high potential for a pandemic ${ }^{[10]}$. Not with standing the high transmission effectiveness of SARS-CoV-2, the progression and comfort of worldwide travel could additionally upgrade its overall spread ${ }^{[9]}$.

Clinical presentation: Starting at 10 February 2020, just three generally enormous scope case considers have completely exhibited the clinical highlights of patients with pneumonia brought about by SARS-CoV-2 (SARS-CoV-2 pneumonia) in Wuhan ${ }^{[11,12,7]}$. In this, we abridge the clinical indications of the 278 pooled patients with SARS-CoV-2 pneumonia which is likewise alluded to as novel coronavirus pneumonia or then again Wuhan pneumonia. The entirety of the patients were grown-ups more seasoned than 18 years old and guys involved $61.9 \%$ of the patients $(n=172)$. An ongoing report in Beijing detailed that 2 of the 13 patients with SARS-CoV-2 pneumonia were kids matured between $2-15$ years $^{[5]}$. Starting at 10 February 2020, in excess of 20 pediatric cases have been accounted for in China, 10 of whom were recognized in Zhejiang Province and were in the age scope of 112 days to 17 years ${ }^{[11]}$. Among grown-up patients, cardiovascular sickness and hypertension were the most well-known hidden ailments, trailed by diabetes mellitus. Fever was the most well-known side effect $(92.8 \% ; \mathrm{n}=258)$, trailed by cough (69.8\%; $\mathrm{n}=194)$, dyspnoea $(34.5 \%$; $\mathrm{n}=96)$, myalgia $(27.7 \% ; n=77)$, cerebral pain $(7.2 \% ; n=20)$ and loose bowels $(6.1 \% ; n=17)$. Rhinorrhoea was noted in just $4.0 \%{ }^{[13]}$, a sore throat in $5.1 \%{ }^{[13]}$ and pharyngalgia in $17.4 \%^{[7]}$ of patients with applicable clinical data. Most patients had a typical white platelet tally, yet $56.8 \%$ ( $\mathrm{n}=$ 158) of patients had leukopenia. In one examination, patients requiring escalated care were essentially more seasoned and bound to have fundamental sicknesses ${ }^{[7]}$, however, another study demonstrated various discoveries $^{[12]}$. As per two investigations, patients admitted to the emergency unit were more probable to have dyspnoea than non-ICU patients ${ }^{[12,7]}$. Among the 13 patients with SARS-CoV-2 pneumonia detailed in Beijing, 12 (92.3\%) had fever with a mean length of 1.6 days before hospitalization ${ }^{[5]}$. Different side effects included cough (46.3\%), upper aviation route clog (61.5\%), myalgia $(23.1 \%)$ and migraine $(23.1 \%)^{[5]}$. Despite the fact that a portion of the epidemiological attributes were recognized, extensive vulnerabilities are as yet present and extra investigations are required with definite data from affirmed cases ${ }^{[14]}$.

\section{CONCLUSION}

Although, Coronaviruses have widely spread and harvested the lives of thousands of people, they are not well understood and vital information remain to be generated.

\section{REFERENCES}

01. Masters, P.S., 2006. The molecular biology of coronaviruses. Adv. Virus Res., 66: 193-292.

02. Robson, B., 2020. Computers and viral diseases. Preliminary bioinformatics studies on the design of a synthetic vaccine and a preventative peptidomimetic antagonist against the SARS-CoV-2 (2019-nCoV, COVID-19) coronavirus. Comput. Biol. Med., Vol. 119, 10.1016/j.compbiomed. 2020.103670

03. Lai, C.C., T.P. Shih, W.C. Ko, H.J. Tang and P.R. Hsueh, 2020. Severe acute respiratory syndrome coronavirus 2 (SARS-CoV-2) and corona virus disease-2019 (COVID-19): The epidemic and the challenges. Int. J. Antimicrob. Agents, Vol. 55, No. 3.

04. Carlos, W.G., C.S.D. Cruz, B. Cao, S. Pasnick and S. Jamil, 2020. Novel Wuhan (2019-nCoV) coronavirus. Am. J. Respir. Crit. Care Med., 201: P7-P8.

05. Chang, D., M. Lin, L. Wei, L. Xie, G. Zhu, C.S.D. Cruz and L. Sharma, 2020. Epidemiologic and clinical characteristics of novel coronavirus infections involving 13 patients outside Wuhan, China. JAMA., Vol. 2020, 10.1001/jama.2020.1623.

06. Li, Q., X. Guan, P. Wu, X. Wang, L. Zhou and Y. Tong et al., 2020. Early transmission dynamics in Wuhan, China, of novel coronavirus-infected pneumonia. N. Engl. J. Med., 382: 1199-1207.

07. Wang, D., B. Hu, C. Hu, F. Zhu and X. Liu et al., 2020. Clinical characteristics of 138 hospitalized patients with 2019 novel Coronavirus-infected pneumonia in Wuhan, China. JAMA., 323: 1061-1069.

08. Zhao, S., Q. Lin, J. Ran, S.S. Musa and G. Yang et al., 2020. Preliminary estimation of the basic reproduction number of novel Coronavirus (2019-nCoV) in China, from 2019 to 2020: A data-driven analysis in the early phase of the outbreak. Int. J. Infect. Dis., 92: 214-217.

09. Biscayart, C., P. Angeleri, S. Lloveras, T.D.S.S. Chaves,P. Schlagenhauf and A.J. RodríguezMorales, 2020. The next big threat to global health? 2019 novel coronavirus (2019-nCoV): What advice can we give to travellers?-Interim recommendations January 2020, from the Latin-American society for Travel Medicine (SLAMVI). Travel Med. Infect. Dis., Vol. 33, 10.1016/j.tmaid.2020.101567 
10. Munster, V.J., M. Koopmans, N. van Doremalen, D. van Riel and E. de Wit, 2020. A novel Coronavirus emerging in China-key questions for impact assessment. N. Engl. J. Med., 382: 692-694.

11. Chen, Z.M., J.F. Fu, Q. Shu, Y.H. Chen and C.Z. Hua et al., 2020a. Diagnosis and treatment recommendations for pediatric respiratory infection caused by the 2019 novel coronavirus. World J. Pediatr., 2020: 1-7.

12. uang, C., Y. Wang, X. Li, L. Ren and J. Zhao et al., 2020. Clinical features of patients infected with 2019 novel Coronavirus in Wuhan, China. Lancet, 395: 497-506.
13. Chen, N., M. Zhou, X. Dong, J. Qu and F. Gong et al., 2020b. Epidemiological and clinical characteristics of 99 cases of 2019 novel Coronavirus pneumonia in Wuhan, China: A descriptive study. Lancet, 395: 507-513.

14. Ryu, S. and B.C. Chun, 2020. Epidemiological characteristics of 2019 novel coronavirus: An interim review. Epidemiol. Health, Vol. 42, 10.4178/epih.e2020006 